\title{
APPROXIMATIONS BY DIFFERENCES OF LOWER SEMICONTINUOUS FUNCTIONS
}

\author{
EDUARD OMASTA
}

\begin{abstract}
A classical theorem of W. Sierpiński, S. Mazurkiewicz and S. Kempisty says that the class of all differences of lower semicontinuous functions is uniformly dense in the space of all Baire-one functions. We show a generalization of this result to more general situations and derive an abstract theorem in the case of a binormal topological space.
\end{abstract}

\section{Introduction}

Nowadays, the approximation of Baire-one functions on the real line by differences of lower semicontinuous functions belongs to a classical theory of real functions. There are several generalizations of this situation. For example, an approximation of approximately continuous functions or an approximation of finely continuous functions in potential theory. In this paper, we concentrate mainly on a unified approach using topological methods allowing an approximation of functions from a class of differences of lower semicontinuous functions.

\section{Preliminaries}

Before proceeding, some notation conventions will be established.

In all what follows, $\mathbb{N}, \mathbb{Z}, \mathbb{R}$ will denote the sets of all natural, integer and real numbers, respectively. The symbol $\mathbb{R}^{n}$ stands for the Euclidean $n$-dimensional space.

A function $f$ is positive if $f \geq 0$. If $f$ is a real function on a set $X$, the symbol $[f>\alpha]$ stands for $\{x \in X: f(x)>\alpha\}$. Similarly, we abbreviate $[f \geq \alpha],[f=\alpha]$, etc.

(C) 2015 Mathematical Institute, Slovak Academy of Sciences. 2010 Mathematics Subject Classification: 54E55, 26A15, 26A21, 31C45, 31C40. Keyw ords: binormal spaces, Baire-one functions, approximately continuous functions, finely continuous functions, density topologies. 
All topological spaces will be considered Hausdorff.

Let $\mathcal{C}(T)$ denote the space of all real-valued continuous functions on a topological space $T$ equipped with the sup-norm $\|\cdot\|$.

Recall that a real-valued function on a topological space $T$ is said to be a Baire-one function if it is a pointwise limit of a sequence of continuous functions on $T$.

\section{Approximation in binormal topological spaces}

We state our main theorem in a general context of bitopological spaces described by J. C. Kelly in [3]. A space $X$ on which two arbitrary topologies $\varrho$ and $\tau$ are defined is called a bitopological space and is denoted by $(X, \varrho, \tau)$.

Topological notions referring to $\tau$-topology will be qualified by prefix $\tau$ to distinguish them from those ones pertaining to topology $\varrho$. A space $(X, \varrho, \tau)$ is said to be binormal if, given a $\varrho$-closed set $A$ and a $\tau$-closed set $B$ with $A \cap B=\emptyset$, there exist a $\tau$-open set $U$ and $\varrho$-open set $V$ such that $A \subset U, B \subset V$ and $U \cap V=\emptyset$. In [3, Theorem 2.7] J. C. K elly proved the following generalization of Urysohn's lemma.

Theorem 3.1. If $(X, \varrho, \tau)$ is binormal then, given a $\tau$-closed set $F$ and a $\varrho$-closed set $H$ with $F \cap H=\emptyset$, there exists a function $g$ on $X$ such that

(a) $g=0$ on $F, g=1$ on $H$ and $0 \leq g \leq 1$ on $X$,

(b) $g$ is upper $\varrho$-semicontinuous and lower $\tau$-semicontinuous.

LEMma 3.2. Let $(X, \varrho, \tau)$ be a binormal topological space, $F_{\tau}$ a $\tau$-closed set and $G$ a $G_{\delta}$-set in the topology $\varrho$. If $F_{\tau} \subset G$, then there exists a lower $\tau$-semicontinuous and upper $\varrho$-semicontinuous bounded positive function $f$ on $X$ such that

$$
F_{\tau} \subset[f=0] \subset G .
$$

Pro of. Let $G_{n}$ be $\varrho$-open sets such that

$$
G=\bigcap_{n=1}^{\infty} G_{n}
$$

and, for each $n \in \mathbb{N}$, let $f_{n}$ be a function from Theorem 3.1 for $F=F_{\tau}$ and $H=X \backslash G_{n}$. Then, the function $f:=\sum 2^{-n} f_{n}$ satisfies our assertion.

Now, consider a topological space $(X, \varrho)$ which is also equipped with some fine topology $\tau$, that is, any topology which is finer than the original topology $\varrho$. This concept is a wide generalization of a concrete example of the fine topology arising in potential theory. 


\section{APPROXIMATIONS BY DIFFERENCES OF LOWER SEMICONTINUOUS FUNCTIONS}

Theorem 3.3. Assume that $\tau$ is a fine topology on a topological space $(X, \varrho)$ and that $(X, \varrho, \tau)$ is a binormal space. Then any real $\varrho$-Baire-one and $\tau$-continuous function on $X$ can be uniformly approximated by differences of two positive $\tau$-continuous and lower $\varrho$-semicontinuous functions.

Proof. Assume that $f$ is a real $\varrho$-Baire-one and $\tau$-continuous function on $X$ and $k$ is an integer. Since $f$ is $\varrho$-Baire-one, the set $[k-1<f<k+1]$ is $F_{\sigma}$ in the topology $\varrho$ (cf. [5, 3.A.1]) and since $f$ is $\tau$-continuous, the set $[k-2<f<k+2]$ is $\tau$-open. Thus, applying Lemma 3.2 to the complements of these sets, there exists $\tau$-continuous and upper $\varrho$-semicontinuous bounded positive function $w_{k}$ on $X$ such that

$$
[k-1<f<k+1] \subset\left[w_{k}>0\right] \subset[k-2<f<k+2] .
$$

Moreover, by multiplying $w_{k}$ by an appropriate constant, we obtain

Set

$$
0 \leq w_{k} \leq \frac{1}{3} e^{-k^{2}}
$$

$$
u(x):=\sum_{k \in \mathbb{Z}} w_{k}(x) \quad \text { and } \quad v(x):=\sum_{k \in \mathbb{Z}} e^{k} w_{k}(x) .
$$

The sums are finite for every $x \in X$ since $w_{k}(x)$ can be strictly positive only for $f(x)-2<k<f(x)+2$. Thus, $u, v$ are $\tau$-continuous and upper $\varrho$-semicontinuous and $0<u(x)<1,0<v(x)<1$ for every $x \in X$. Moreover,

$$
\mathrm{e}^{f(x)-2} \sum_{k \in \mathbb{Z}} w_{k}(x)<\sum_{k \in \mathbb{Z}} \mathrm{e}^{k} w_{k}(x)<\mathrm{e}^{f(x)+2} \sum_{k \in \mathbb{Z}} w_{k}(x),
$$

and so,

for any $x \in X$. The functions

$$
\mathrm{e}^{f(x)-2}<\frac{v(x)}{u(x)}<\mathrm{e}^{f(x)+2}
$$

$$
s(x):=-\log u(x) \quad \text { and } \quad t(x):=-\log v(x)
$$

are positive $\tau$-continuous and lower $\varrho$-semicontinuous and, moreover,

$$
|f(x)-(s(x)-t(x))|<2,
$$

for every $x \in X$. From this, the assertion easily follows.

In general, this approximation cannot be done by differences of bounded lower $\varrho$-semicontinuous functions even for bounded $\varrho$-Baire one and $\tau$-continuous functions.

Proposition 3.4. Let $\varrho$ be Euclidean metric topology on $\mathbb{R}$ and $\tau$ be a fine topology on $(\mathbb{R}, \varrho)$ such that $(\mathbb{R}, \varrho, \tau)$ is binormal and every countable subset of $\mathbb{R}$ is $\tau$-closed. Then, there exists a bounded $\varrho$-Baire-one and $\tau$-continuous 


\section{EDUARD OMASTA}

function $h$ on $\mathbb{R}$ such that if $f, g$ are lower $\varrho$-semicontinuous functions and $\|h-(f-g)\|<1$, then $f$ and $g$ are unbounded.

Proof. Let

$$
A_{1}:=\left\{n+\frac{1}{2}: n \in \mathbb{Z}\right\} .
$$

For every $x \in A_{1}$, there exists an $\varepsilon_{x}>0$ such that $\left(x-\varepsilon_{x}, x+\varepsilon_{x}\right) \cap A_{1}=\{x\}$. We define

$$
A_{2}:=\bigcup_{x \in A_{1}}\left\{x-\frac{\varepsilon_{x}}{2 n}: n \in \mathbb{N}\right\}
$$

and since every point of $A_{2}$ is isolated, the definition continues by induction. Hence, the set $A_{n}$ consists of limit points of $A_{n+1}$ for every $n \in \mathbb{N}$. Let

$$
B_{n, i}:=A_{i} \cap(n, n+1) \quad \text { for } \quad n \in \mathbb{N} \text { and } i \in[1, n] .
$$

The sets

$$
\begin{aligned}
& X:=\bigcup_{n=1}^{\infty} \bigcup_{1 \leq 2 i-1 \leq n} B_{n, 2 i-1}, \\
& Y:=\bigcup_{n=2}^{\infty} \bigcup_{2 \leq 2 i \leq n} B_{n, 2 i}
\end{aligned}
$$

are countable and thus $\tau$-closed. Moreover, they are scattered and thus, $G_{\delta}$ (cf. in [8, Theorem A.3]). Using Lemma 3.2, there exist $\tau$-continuous and upper $\varrho$-semicontinuous functions $\varphi_{1}$ and $\varphi_{2}$ such that $\varphi_{1}(x)=0$ for $x \in X, \varphi_{1}(x)>0$ otherwise, and $\varphi_{2}(x)=0$ for $x \in Y, \varphi_{2}(x)>0$ otherwise. Thus, for the $\varrho$-Baire-one and $\tau$-continuous function

$$
h(x):=\frac{5 \varphi_{2}(x)}{\varphi_{1}(x)+\varphi_{2}(x)},
$$

we have

$$
h(x)=\left\{\begin{array}{lll}
5 & \text { for } & x \in X, \\
0 & \text { for } & x \in Y,
\end{array}\right.
$$

and $0<h(x)<5$ otherwise.

Let $f, g$ are lower $\varrho$-semicontinuous functions such that $\|h-(f-g)\|<1$. Pick an arbitrary $n \in \mathbb{N}$ and choose $x \in B_{2 n, 1}$. Then, $h(x)=5$, so, $4<f(x)-g(x)<6$, and hence, $f>g(x)+4$ on some neighbourhood of $x$. Consequently, $f(y)>$ $g(x)+4$ for some $y \in B_{2 n, 2}$. Since $h(y)=0$, we have $g(y)>f(y)-1>g(x)+3$ and thus, $g>g(x)+3$ on some neighbourhood of $y$. Therefore, $g(z)>g(x)+3$ for some $z \in B_{2 n, 3}$. Thus, we have

$$
f(z)>g(z)+4>g(x)+7>f(x)+1
$$


and by repeating this procedure, we get an existence of $w \in B_{2 n, 2 n-1}$ such that $f(w)>f(x)+n-1$. It follows that the function $f$ is unbounded, and therefore, $g$ is unbounded, too.

Remark 3.5. There are many topologies, where approximations by differences of lower semicontinuous functions play an important role. Let us mention the classical fine topology in potential theory on $\mathbb{R}^{n}$ defined as the smallest topology on $\mathbb{R}^{n}$ for which all Newtonian potentials are continuous. Since every Newtonian potential on $\mathbb{R}^{n}$ is approximately continuous, the classical fine topology on $\mathbb{R}^{n}$ is coarser than the ordinary density topology on $\mathbb{R}^{n}$. This topology is finer than the Euclidean topology. The existence of discontinuous Newtonian potentials implies that this topology is properly finer than the Euclidean one. The Euclidean topology and the classical fine topology makes $\mathbb{R}^{n}$, a bitopological space. Moreover, any finely continuous function is a Baire-one function on $\mathbb{R}^{n}$. Hence, we may apply again the general Theorem 3.3. A relevant material can be found in [5].

Another example of a fine topology on $\mathbb{R}^{n}$ arises from a nonlinear potential theory. Let $p \in(1, n)$. Consider the following $p$-Laplace equation

$$
\operatorname{div}\left(|\nabla u|^{p-2} \nabla u\right)=0,
$$

where solutions have to be understood in a weak sense. When $p=2$, this equation reduces to the classical Laplace equation. The $p$-fine topology is generated by the collection of all $p$-superharmonic functions on $\mathbb{R}^{n}$ and together with the Euclidean topology forms a binormal topological space (cf. in [2, Theorem 3.4]).

\section{Approximation theorem in $\mathbb{R}^{n}$}

Let $\varrho$ be the Euklidean metric on $\mathbb{R}^{n}$ and let $\tau$ be the discrete topology on $\mathbb{R}^{n}$. The bitopological space $\left(\mathbb{R}^{n}, \varrho, \tau\right)$ is clearly binormal since every $\varrho$-closed set is $\tau$-open. Moreover, every real function on $\mathbb{R}^{n}$ is $\tau$-continuous. So, from Theorem 3.3, we have the following proposition as an immediate consequence.

Proposition 4.1. Any Baire-one function on $\mathbb{R}^{n}$ can be uniformly approximated by differences of two positive lower semicontinuous functions.

This proposition goes back to S. Mazurkiewicz [6] and W. Sierpiński [7] (they proved the result for bounded functions) and to $\mathrm{S}$. K e m p is t y [4] (who dropped the condition of boundedness).

Remark 4.2. The assertion that there exist bounded Baire-one functions, which cannot be approximated by differencies of bounded lower semicontinuous functions, belongs to mathematical folklore. It is an obvious consequence of Proposition 3.4, however, more can be said in the case of real line. In the next proposition, we present a simple example of a bounded Baire-one function which is even 


\section{EDUARD OMASTA}

a difference of lower semicontinuous functions though it cannot be approximated by differencies of bounded lower semicontinuous functions.

Proposition 4.3. There exists a bounded Baire-one function $h$ on $\mathbb{R}$ which is a difference of two lower semicontinuous functions such that if $f, g$ are lower semicontinuous functions on $\mathbb{R}$ and $\|h-(f-g)\|<1$, then $f$ and $g$ are unbounded.

Proof. Let $\left\{A_{i}\right\}$ be a sequence of sets defined in the proof of Proposition 3.4. Since the set $A_{n}$ consists of limit points of $A_{n+1}$ for every $n \in \mathbb{N}$, the sets

$$
K_{0}:=\emptyset \quad \text { and } \quad K_{n}:=\bigcup_{k=1}^{n} A_{k}
$$

are closed for every $n \in \mathbb{N}$.

For $n \in \mathbb{N}$, denote

and

$$
X_{n}:=A_{n} \backslash[-n, n]
$$

Then,

$$
Y_{n}:=[-n, n] \backslash\left([-(n-1), n-1] \cup K_{n-1}\right) .
$$

$$
\mathbb{R}=\{0\} \cup \bigcup_{n=1}^{\infty}\left(X_{n} \cup Y_{n}\right),
$$

where all the sets in this union are pairwise disjoint.

Now, define the function $\varphi$ as follows: Given $x \in \mathbb{R} \backslash\{0\}$, there exists $n \in \mathbb{N}$ such that $x \in X_{n} \cup Y_{n}$. Set $\varphi(x):=n$. Further, set $\varphi(0):=1$.

Analogously, for $n \in \mathbb{N}$, set

$$
U_{n}:=\left(A_{2 n-1} \cup A_{2 n}\right) \backslash[-2 n, 2 n]
$$

and

Then,

$$
V_{n}:=[-2 n, 2 n] \backslash\left([-(2 n-2), 2 n-2] \cup K_{2 n-2}\right) .
$$

$$
\mathbb{R}=\{0\} \cup \bigcup_{n=1}^{\infty}\left(U_{n} \cup V_{n}\right),
$$

where all the sets in this union are pairwise disjoint.

Define the function $\psi$ as follows: Given $x \in \mathbb{R} \backslash\{0\}$, there exists $n \in \mathbb{N}$ such that $x \in U_{n} \cup V_{n}$. Set $\psi(x):=2 n$. Further, set $\psi(0):=2$.

The functions $\varphi$ and $\psi$ are obviously lower semicontinuous and the function $h:=5(\psi-\varphi)$ is a bounded Baire-one function on $\mathbb{R}$.

Let $f, g$ be lower semicontinuous functions such that $\|h-(f-g)\|<1$. Pick an arbitrary $n \in \mathbb{N}$ and choose $x \in A_{1} \cap(2 n+1,2 n+2)$. Since $h(x)=5$, we have $4<f(x)-g(x)<6$, and thus, $f>g(x)+4$ on some neighbourhood of $x$. Since $x \in A_{1}$ is a limit point of $A_{2}$, there exists $y \in A_{2} \cap(2 n+1,2 n+2)$ such that $f(y)>g(x)+4$. Since $h(y)=0$, we have $g(y)>f(y)-1>g(x)+3$ and so, 
$g>g(x)+3$ on some neighbourhood of $y$. Thus, there exists $z \in A_{3} \cap(2 n+1,2 n+2)$ with $g(z)>g(x)+3$. Hence,

$$
f(z)>g(z)+4>g(x)+7>f(x)+1 .
$$

By induction, there exists $w \in A_{2 n+1} \cap(2 n+1,2 n+2)$ such that $f(w)>f(x)+n$. Thus, the functions $f$ and $g$ are unbounded.

\section{Density topologies}

The ordinary density topology on $\mathbb{R}^{n}$ is defined as the coarsest topology making all the approximately continuous functions on $\mathbb{R}^{n}$ continuous or, equivalently, it is formed by a collection of Lebesgue measurable sets on $\mathbb{R}^{n}$ having all their points as a point of ordinary density.

Let $\varrho$ be the Euklidean metric on $\mathbb{R}^{n}$ and let $\tau$ be the ordinary density topology on $\mathbb{R}^{n}$. Then the bitopological space $\left(\mathbb{R}^{n}, \varrho, \tau\right)$ is binormal. The proof can be found in [1] (cf. also in [5, Section 6.D ]). Since a function is approximately continuous if and only if it is $\tau$-continuous and since any approximately continuous function on $\mathbb{R}^{n}$ is a Baire-one function, according to Theorem 3.3. we immediately get the following proposition.

Proposition 5.1. Any approximately continuous function on $\mathbb{R}^{n}$ can be uniformly approximated by differences of positive approximately continuous and lower semicontinuous functions.

Remarks 5.2. (a) In [9], S. V a n ěč e k proved this proposition for the case of bounded approximately continuous functions. His proof is based on a simple generalization of the Stone-Weierstrass theorem.

Moreover, he showed that there exists a bounded approximately continuous function $\mathrm{h}$ on $\mathbb{R}$ such that if $f, g$ are positive lower semicontinuous functions on $\mathbb{R}$ and $\|h-(f-g)\|<1$, then $f$ and $g$ are unbounded.

(b) The density topology is an important example of a more general concept of abstract density topologies. Given a measure space $(X, \mathcal{S}, \mu)$, where $\mu$ is a $\sigma$-finite and complete, a topology $\tau$ is said to be an abstract density topology on $(X, \mathcal{S}, \mu)$ if the class of $\mu$-null sets coincides with the class of $\tau$-closed and $\tau$-nowhere dense sets and, moreover, a set $M$ belongs to $\mathcal{S}$ if and only if $M$ has the $\tau$-Baire property.

Given an abstract density topology $\tau$ on $(X, \mathcal{S}, \mu)$, sometimes another (original, coarser) topology $\varrho$ is considered on $X$. It would be interesting to examine when $(X, \varrho, \tau)$ forms a binormal topological space.

Acknowledgements. I would like to thank the referee for valuable comments that improved this paper. 


\section{EDUARD OMASTA}

\section{REFERENCES}

[1] GOFFMAN, C.-NEUGEBAUER, C.-NISHIURA, T.: Density topology and approximate continuity, Duke Math. J. 28 (1961), 497-505.

[2] HEINONEN, J.-KILPELÄINEN, T.-MALÝ, J.: Connectedness in fine topologies, Ann. Acad. Sci. Fenn. Math. 15 (1990), 107-123.

[3] KELLY, J. C.: Bitopological spaces, Proc. Lond. Math. Soc. 13 (1963), 71-89.

[4] KEMPISTY, S.: Sur l'approximation des fonctions de première classe, Fund. Math. 2 (1921), 131-135.

[5] LUKEŠ, J.-MALÝ, J.-ZAJÍČEK, L.: Fine topology methods in real analysis and potential theory. Lecture Notes in Math., Vol. 1189, Springer-Verlag, 1986.

[6] MAZURKIEWICZ, S.: Sur les fonctions de classe 1, Fund. Math. 2 (1921), 28-36.

[7] SIERPIŃSKI, W.: Demonstration d'un théorème sur les fonctions de première classe, Fund. Math. 2 (1921), 37-40.

[8] THOMSON, B. S.: Symmetric Properties of Real Functions, in: Monogr. Textbooks Pure Appl. Math., Vol. 183, CRC Press, 1994.

[9] VANĚČEK, S.: On uniform approximation of bounded approximately continuous functions by differences of lower semicontinuous and approximately continuous ones, Czechoslovak Math. J. 110 (1985), 28-30.

Received January 19, 2015

\author{
Eduard Omasta \\ Department of Mathematics \\ Pedagogical Faculty \\ Catholic University \\ Hrabovská cesta 1 \\ SK-034-01 Ružomberok \\ SLOVAKIA
}

E-mail: Eduard.Omasta@ku.sk 